MEI, II, Vol. 2, nº 2, pág. 67

MEI

II, vol. 2

$\mathrm{n}^{0} 2$

\title{
La Biblioteca General de la Diputación de Valencia: evolución histórica
}

\author{
Ana María Reig Ferrer \\ Biblioteca del MuVIM
}

\section{Resumen}

La Diputación de Valencia, a lo largo de su historia, creó instituciones que potenciaron la cultura valenciana, además de apoyar y promocionar el libro valenciano, consciente de su papel como impulsora y difusora de su identidad local. La Biblioteca Provincial, creada en 1926, pretendió ser un espacio de difusión cultural, pero la falta de un espacio propio y la diversificación de proyectos impidieron que pudiera ser el germen de la futura biblioteca nacional valenciana.

\section{Palabras clave}

Diputación Provincial de Valencia; Política cultural; Biblioteca de la Diputación de Valencia.

The Library of the Provincial Council Valencia: historical evolution

\section{Abstract}

The Diputación de Valencia, throughout its history, has created institutions that fostered the valencian culture, in addition to supporting and promoting the valencian book, aware of its role as a promoter and disseminator of local identity. The provincial library, established in 1926, claimed to be an area of cultural diffusion, but the lack of a proper space and the diversification of projects prevented it becoming the seed of the future national library of Valencia.

\section{Keywords}

Diputación Provincial of Valencia; Cultural policy; Diputación of Valencia’s library.

El objetivo de este trabajo es conocer la evolución histórica de la Biblioteca General de la Diputación de Valencia, cómo se formó y el porqué de los fondos que la integran. Se trata de una de las bibliotecas más antiguas de la ciudad de Valencia, dependiente de una administración pública con carácter territorial y cultural propio. Actualmente forma parte de la biblioteca del Museu Valencià de la Il·lustració i de la ModernitatMuVIM.

\section{Contexto histórico de la Diputación Provincial de Valencia}

Las diputaciones tienen su origen en las Cortes de Cádiz pero su consolidación como entidades locales, de ámbito provincial, no se produjo hasta bastantes años después. Estas instituciones, al igual que otras, no fueron ajenas a los cambios políticos del primer tercio del siglo XIX.

En 1814, con la llegada de Fernando VII al poder, las diputaciones fueron suprimidas y reaparecieron durante el Trienio Liberal (1820-1823), 
momento en el cual aflora otra diputación, la de Xàtiva (1822-1823), al dividirse el territorio valenciano en cuatro provincias: Castellón, Valencia, Xàtiva y Alicante (Decreto de las Cortes de 27 de enero de 1822 y R. O. de 29 de marzo). La vuelta de Fernando VII, en 1823, con la ayuda europea de los Cien mil hijos de San Luis, daba fin al Trienio Liberal y volvían a desaparecer las diputaciones. Habría que esperar la muerte del rey, en 1833, para que las diputaciones se restablecieran definitivamente. Así el R. D. de 30 de noviembre de 1833, sobre división territorial definitiva, acordaba la reducción a tres provincias: Castellón, Valencia y Alicante.

La Diputación de Valencia celebró su primera sesión el 17 de enero de 1836, presidida por el gobernador civil. Las atribuciones conferidas eran, además del control y supervisión de la administración municipal, competencias en materia de fomento de la agricultura, industria, montes, minas, carreteras y caminos vecinales, instrucción pública y Bellas Artes; a las que se sumaron las benéficas, tras la ley de Beneficencia de 1849 y la supresión de las Juntas de Beneficencia en 1868. De ahí que la Diputación de Valencia asumiera establecimientos benéficos como la Casa de la Misericordia, la Casa de la Beneficencia y el Hospital General, así como los dependientes de este último, que eran el Teatro principal y la Plaza de Toros.

Estas competencias cambiarán a partir del estatuto municipal de 1924, que otorgaba más autonomía a los ayuntamientos, y el provincial de 1925; pero hasta la Constitución Española de 1978 y la promulgación del Estatuto de Autonomía en 1982, que han limitado sus competencias, podemos considerar que las diputaciones fueron durante 150 años unas instituciones administrativas, de carácter local, de gran importancia a nivel político, educativo, cultural y social.

Centrándonos en el tema educativo-cultural, que es el que nos interesa, debemos mencionar que las corrientes regeneracionistas, extendidas en el último cuarto del siglo XIX, intentaron concienciar a la sociedad de la importancia de la educación como motor de transformación social, capaz de formar hombres y mujeres preparados para mejorar la sociedad. En este contexto habría que situar los postulados pedagógicos del krausismo que dieron lugar a instituciones educativas privadas como la Institución Libre de Enseñanza (ILE) y otras afines.

El siglo XX comenzó con una serie de reformas en el campo de la educación y fueron hombres cercanos a la Institución Libre de Enseñanza (ILE) quienes llevaron a cabo estas reformas. En 1900 se creó el Ministerio de Instrucción Pública y Bellas Artes y se impulsó el fomento de la educación siguiendo un texto de Manuel Cossío, que no era más que una síntesis del programa de reformas de los institucionalistas. Otros colaboradores de la reforma fueron Rafael Altamira, Álvaro Figueroa y Amalio Gimeno, todos ellos cercanos a la ILE.

La Diputación de Valencia no fue ajena a estos postulados krausistas y dentro de ese espacio temporal habría de situar la creación de las primeras bibliotecas de la Diputación de Valencia: las escolares, dependientes de los centros educativos de la corporación, como claro apoyo a la enseñanza. En la memoria sobre las bibliotecas existentes en España en el último cuarto del siglo XIX, realizada por Nicolás Díaz y 
Pérez, ya consta la biblioteca de la escuela de niños de la Casa de la Beneficencia (Díaz, N., 1885, p. 56). De ahí que puede considerarse esta biblioteca escolar como la primera biblioteca existente en la Diputación de Valencia.

\section{Creación de la Biblioteca Provincial de la Diputación de Valencia}

La Diputación de Valencia tomaba conciencia de su papel protector y difusor de la cultura valenciana con la creación del Centro de Cultura Valenciana, en 1915, centro que funcionaría bajo su protección y con la colaboración del Ayuntamiento de Valencia. El artículo segundo de sus estatutos decía: «El objeto del Centro de Cultura es cultivar la historia integral de la ciudad de Valencia y de su antiguo Reino, y el fomento de aquellas ramas del saber humano que carecen de corporación o entidad regional dedicadas especialmente a ellas, pudiendo indistintamente usar en sus trabajos las lenguas castellana y valenciana» ${ }^{1}$.

Esta labor quedaría confirmada en 1919 con la creación de la editorial «Acción Bibliográfica Valenciana» que proyectaba publicar obras de carácter histórico valenciano $^{2}$. Tampoco se olvidaba de las obras escritas por valencianos, al menos de aquellos más allegados. Así, ese mismo año, la Diputación adquiría varios ejemplares de la obra El Real Templo Basílica de San Francisco el Grande en la Historia y en las Artes, escrita por el capellán mayor de la basílica José Calabuig Revert, quien había sido antiguo asilado de la Casa de la Misericordia. Esta obra había sido muy bien acogida en los centros artísticos madrileños, especialmente en la Academia de San Fernando.

El hecho de que su autor se hubiera formado en uno de los centros benéficos de la Diputación fue motivo más que suficiente para que la corporación provincial se mostrara satisfecha y acordara «adquirir ejemplares de la citada obra para que figuren en las bibliotecas de la Diputación y Casas de Misericordia y Beneficencia, como testimonio de lo bien que el Sr. Calabuig supo aprovechar las primeras enseñanzas recibidas al calor de esta Corporación». Este hecho podría explicar la existencia de varias colecciones de libros en diferentes dependencias de la corporación.

En 1923 Luís Cebrían Ibor sustituye al Sr. Boix Vila como archivero de la Diputación, y uno de los proyectos que presentaba era formar una biblioteca con los libros y folletos existentes en la corporación. Se trataba de un primer intento de reunir y organizar el material bibliográfico que estaba disperso en diferentes oficinas para formar una biblioteca de temática legislativa de apoyo al personal técnico de la corporación ${ }^{3}$.

Tres años más tarde, un Real Decreto de 6 de febrero de 1926, firmado por el Rey Alfonso XIII, instituía la Fiesta Nacional del Libro en España, señalando su celebración para el día 6 de octubre en conmemoración de la fecha de nacimiento de Cervantes. Años después se trasladaría al 23 de abril tras confirmarse esta fecha como la correcta. 
Coincidiendo con esta celebración, la corporación provincial tomaba conciencia de la importancia que suponía para la vida moderna el acceso a la cultura y lo necesario que era una biblioteca como órgano de difusión de ésta: «se ha iniciado por esta Diputación una Biblioteca, que si bien resulta modesta por la cuantía de sus fondos, reviste marcado interés, así por la materia sobre que versan los ejemplares que la integran, como por contener, muchos de ellos, dedicatorias autógrafas $\rangle^{4}$. Con estas palabras, recogidas en la memoria del año 1925-1926, quedaba instituida la biblioteca provincial. En esta misma memoria se hace referencia a las nuevas adquisiciones ingresadas en la biblioteca y las subvenciones, directas o indirectas, que se establecían para la edición de obras de «interés histórico o artístico regional».

Se había creado la Biblioteca Provincial de la Diputación de Valencia. Una biblioteca que, según se desprende de la memoria del año siguiente, había tenido muy buena acogida por la opinión pública, tanto es así que en tan sólo un año se habían incrementado considerablemente los fondos, tanto por compra como por donación. A partir de ese año, en las memorias de la corporación, son continuas las referencias a los libros que ingresan cada año.

\section{Evolución histórica de la Biblioteca de la Diputación: de Provincial a General}

La corporación, sensibilizada cada vez más de la necesidad de ampliar su biblioteca y de abrirla al público, se planteó buscar un local adecuado. La primera noticia que tenemos la situaba junto al salón de sesiones de la corporación provincial permanente «por no disponerse por ahora de sitio más adecuado». Esta biblioteca, denominada provincial, estaba especializada en temática local: libro valenciano, de tema valenciano y escrito por valencianos. De manera que nos encontramos con dos bibliotecas totalmente diferenciadas: una, la general o provincial, especializada en temas de historia y cultura valenciana y otra, auxiliar del archivo, dependiente de secretaría, con una colección de temática jurídica y administrativa, de la cual se señala en la memoria que, además de libros, cuenta «con una colección de folletos, así como de publicaciones y periódicos oficiales».

El año 1927 fue un año fructífero para la biblioteca provincial. La corporación asumía que el libro y la enseñanza proporcionaban una sólida base para el desarrollo de los pueblos y, siguiendo la labor que estaban realizando en otras provincias españolas, asumía su papel de protectora cultural, abogando no sólo por la adquisición de obras nuevas, sino también antiguas: «desea acrecentar su labor adquiriendo no sólo obras modernas sino también recogiendo bibliotecas dispersas particulares o que salgan al mercado».

Un año después, debido a la importancia que estaba consiguiendo la biblioteca, el archivero-bibliotecario realizó un inventario general y una valoración de los fondos que la integraban, estableciéndose a partir de entonces un libro registro de entrada. En el año 1928 se contabilizaron 259 libros nuevos, muchos de ellos donaciones de particulares y de colectivos que habían recibido apoyo económico de la corporación. 
Las referencias a colaboraciones entre la institución y los autores son continuas: «Durante el pasado año [1928] han ido aumentando los fondos de la Biblioteca de la Diputación, merced a los libros que han sido regalados a la Corporación; y los que ésta ha adquirido, como medio indirecto, de subvencionar o auxiliar al autor» ${ }^{5}$. De lo que se deduce que la Diputación, además de subvencionar los estudios de temática valenciana, a través de la creación de premios como el concurso anual de «Manuales sobre aspectos de la vida valenciana», también ayudaba a los autores y editores valencianos, potenciando tanto la cultura valenciana como la industria editorial.

En la Memoria de 1930 se puntualiza: «Esta Diputación tiene una Biblioteca que, aunque se halla en período de formación, reviste alguna importancia por la calidad e interés bibliográfico de algunos de los ejemplares que figuran en los fondos que la constituyen» ${ }^{6}$. Ese mismo año sufragaba la impresión de la obra La Diputación de la Generalidad del Reino de Valencia, de Francisco Martínez Aloy (Valencia, 1930), y subvencionaba otras. También adquirió obras, para destinarlas a diferentes bibliotecas, como los 300 ejemplares de la obra Els Furs de Valençia, que adquirió en 1931 con destino a los ayuntamientos de la provincia, escuelas públicas y asilos de beneficencia.

En 1932 no sólo adquiría libros con la finalidad de enriquecer «su ya nutrida Biblioteca» sino que, además, siguiendo con su obra cultural concedía lotes de libros a diferentes entidades y potenciaba la creación de bibliotecas públicas municipales, haciéndose eco del Decreto del Ministerio de Instrucción Pública, de 13 de junio de 1932, por el cual podían solicitar bibliotecas municipales aquellos pueblos que no tuvieran biblioteca pública del Estado. La Comisión Gestora de la Diputación de Valencia publicó un anuncio en el Boletín Oficial de la Provincia con el fin de estimular a los pueblos para que aprovecharan esa iniciativa.

A primeros de la década de los años treinta, la Diputación contaba ya con tres importantes bibliotecas: la Biblioteca Provincial de la Diputación (que era la general), creada en 1926; la del Servicio de Investigación Prehistórica (SIP) que se había ido formando a partir de la creación de este servicio en 1927, y la del Centro de Estudios Económicos Valencianos, creado en 1929 y que pronto contaría con una importante biblioteca y archivo, centro que se convertiría en 1948 en el Instituto Valenciano de Economía (IVE). Estas dos últimas bibliotecas se especializaron en la temática propia del centro al que servían, con la finalidad de apoyar sus investigaciones. La del SIP sigue creciendo y es la que conocemos actualmente como la Biblioteca del Museo de Prehistoria de Valencia y la del IVE actualmente en la Biblioteca Valenciana.

Tras la guerra civil, la Diputación consideraba que la biblioteca era un órgano difusor de la cultura patria, necesario para ayudar a la nueva reconstrucción de España, y consignaba en el presupuesto de 1941 la cantidad de 5.000 pesetas para reorganizar y acrecentar los fondos de la misma.

En 1947, en la sesión del Pleno de 25 de abril, se creaba la Institución Alfonso el Magnánimo, la cual se perfilaba como una entidad encargada de atender las aspiraciones culturales de la corporación provincial. Nacía con el nombre de 
Institución de Estudios Valencianos Alfonso el Magnánimo. La propuesta de creación se debió a Felipe $\mathrm{M}^{\mathrm{a}}$ Garín Ortiz de Taranco, diputado ponente de Cultura, Enseñanza y Bellas Artes, y apoyada por el entonces presidente de la Diputación, Adolfo Rincón de Arellano. El reglamento de esta Institución fue aprobado en la sesión plenaria celebrada el 19 de febrero de 1948, y en él se establecía que los fines de la misma eran:

«Orientar y coordinar la investigación y estudios de naturaleza científica, literaria y artística en la provincia de Valencia, preferentemente en todos aquellos asuntos de carácter e interés local. Coordinar, igualmente, la labor de los distintos organismos culturales valencianos, sirviendo, previa su anuencia, de nexo entre los mismos, con el sólo objeto del más provechoso estímulo. Establecer y mantener la adecuada relación, mediante concierto, con los organismos nacionales de cometido similar, en aquellas materias que cultive esta Institución. Establecer y mantener asimismo relaciones con las instituciones culturales, de carácter general, de las otras provincias de la Región. Asesorar a la Diputación en materia cultural comprendida dentro de la esfera de esta Institución» (Gabarda, V., 1997).

Con la creación de esta institución la Diputación pretendía constituirse como una entidad que recogiera las manifestaciones artísticas y culturales del pueblo valenciano. La prensa de la época se hacía eco de la noticia y el 1 de abril de 1948 aparecía en el periódico Levante el titular «La obra cultural de la Diputación de Valencia» en la que se plasmaban interesantes manifestaciones del presidente de la Diputación, el Sr. Rincón de Arellano.

La Institución Alfons el Magnànim nacía como un instituto dependiente del Consejo Superior de Investigaciones Científicas y con un delegado técnico y bibliotecario de la misma, Arturo Zabala López, quien ocuparía el cargo hasta 1980. El Sr. Zabala era también el archivero-bibliotecario de la Diputación de Valencia, de ahí que las adquisiciones e intercambios de la Institución Alfonso el Magnánimo acabaron formando y ampliando la colección de la biblioteca provincial.

En 1952 la corporación provincial adquiría una de las más importantes colecciones españolas sobre Miguel de Cervantes: la que se denominaría «Colección cervantina». Fue Francisco Martínez y Martínez (1866-1946), un bibliófilo especialista en la obra de Cervantes, quien reunió a lo largo de su vida una importante biblioteca ${ }^{7}$. Esta colección se incorporó a la biblioteca provincial pero formando una colección independiente que debía seguir nutriéndose de nuevas adquisiciones de obras y estudios de Cervantes.

Francisco Martínez y Martínez, nacido en Altea en 1866, estudió Derecho en la Universidad de Valencia y proyectó su vida profesional en actividades culturales: fue miembro de la Sociedad Económica de Amigos del País, de Lo Rat Penat y del Centro de Cultura Valenciana; realizó y publicó estudios sobre folklore, historia y bibliografía de Valencia, pero por lo que fue más conocido en España y en el extranjero fue por sus conocimientos de la vida y obra de Miguel de Cervantes. Su pasión por el mundo 
cervantino hizo que a lo largo de su vida reuniera una importante colección, formada por 1.700 volúmenes, entre Quijotes, Novelas Ejemplares, Comedias, Entremeses, etc., entre las que cabe destacar primeras ediciones, como la primera edición valenciana del Quijote, en 1605, de Pedro Patricio Mey.

Esta colección fue adquirida, por compra, a sus herederos por 600.000 pesetas. El artífice de la gestión fue D. Arturo Zabala y el hecho de fuera el archiverobibliotecario y también director del Instituto de Literatura de la Institución Alfonso el Magnánimo hizo que la biblioteca fuera a la vez sede de dicho instituto y centro de reunión de expertos filólogos, como Sanchis Guarner. Esto hizo que a la biblioteca se le diera una orientación literaria y filológica, de ahí la existencia de un buen número de obras de estas materias, entre las que cabe destacar la colección de obras de la Biblioteca de Autores Españoles (BAE).

La Diputación estaba ubicada en el denominado, erróneamente, Palacio del Temple, nombre con el que es conocido el Sacro Convento de la Orden de Santa María de Montesa. Este edificio fue construido para albergar a los religiosos de esta orden cuando el terremoto de 1748 destruyó el castillo de Montesa. Tras la desamortización, el convento fue ocupado por las oficinas provinciales: Diputación y Gobierno Civil, cuyas competencias no estaban claramente diferenciadas en sus orígenes (el gobernador civil era el presidente de la Diputación), pero con el paso del tiempo éstas fueron diferenciándose y cada una de estas instituciones deseaba tener su propia sede.

La Diputación de Valencia siempre deseó ocupar el antiguo Palacio de la Generalitat, edificio construido a principios del siglo XV para albergar la Casa de la Diputación del general del Reino de Valencia, organismo foral encargado de recaudar las generalidades, cantidades con las que el rey sufragara sus campañas militares. Este edificio estaba ocupado por la Audiencia de Valencia y durante años la Diputación lo estuvo reclamando hasta que lo consiguió a mediados del siglo XX.

Con la reintegración de la corporación a su añorada sede, en 1952, se podía conseguir un mejor emplazamiento para la biblioteca. La Comisión de cultura e investigaciones científicas de la Diputación así lo consideraba y nombraba a los señores diputados Juan Tormo Cervino, presidente de la comisión de cultura, y a Vicente Noguera Espinosa de los Monteros, Marqués de Cáceres, como vocal de Bellas Artes, para que formularan el proyecto. Era junio de 1953 y en diciembre se elevaba una Moción a la Comisión de Cultura por la cual se reconocía el patrimonio bibliográfico «como el exponente de la manifestación cultural y científica y que dicho manantial debía ponerse al alcance del estudioso, el investigador o simplemente el lector con afán de instruirse». Quedaba abierta una nueva vía, la de situar el patrimonio bibliográfico al alcance del ciudadano y para ello era necesario encontrar un local que reuniera unas buenas condiciones para instalar la biblioteca, ponerla en funcionamiento y facilitar su acceso. 
Unos años después, por Decreto $\mathrm{n}^{\circ} 337$ de 20 de abril de 1956, el presidente de la Diputación de Valencia, Francisco Cerdá Reig, aceptaba la propuesta de la Comisión de cultura e investigaciones científicas y acordaba:

"Disponer, desarrollando la idea de Presidencia, que por el Sr. Arquitecto Provincial, y en el plazo más breve posible, se estudie y formule presupuesto para la instalación de la Biblioteca Provincial en el Salón Dorado, de forma que se haga compatible con la sala de exposiciones y reuniones, aprovechando en el proyecto, las estanterías existentes en lo que se proyectó para Biblioteca de la Diputación, existente en el piso arquerio de la Generalidad".

Era la primera vez que se concebía una biblioteca destinada al público como complemento de otras actividades culturales de la institución. Esta propuesta no se llevó a efecto y dos años después se volvía a retomar el tema. En 1958 surgía un nuevo proyecto: crear una biblioteca especializada en materia legislativa. La idea partía de la Secretaría General de la Diputación quien elevaba su propuesta a la Comisión de Cultura e Investigaciones Científicas.

«Se razona la conveniencia de crear una Biblioteca Técnico Provincial, con una especialización destacada en el campo jurídico de la Administración Pública. Aprobar el Reglamento... para la implantación y funcionamiento de dicha Biblioteca, cuya instalación se llevará a cabo en local adecuado del Palacio de la Generalidad. Y determinar que a la Biblioteca Técnico Provincial, que tan útiles y provechosos servicios ha de representar para quienes dedican sus actividades profesionales a una materia que por la profusión de legislación, exige cada vez mayores conocimientos, se incorpore en principio la Biblioteca Cervantina, para que ocupe el lugar preeminente que demanda su interés bibliográfico universal, viniendo así a ser el primer jalón para la creación en su día de la Biblioteca Pública, tal como lo requiere la importancia de los fondos existentes y el prestigio cultural y buen nombre de la propia Corporación» ${ }^{8}$.

Las razones para justificar la creación de una «biblioteca técnica» eran obvias; el cúmulo de disposiciones legales que se dictaban sobre las mismas materias, las variaciones y modificaciones, y las nuevas disposiciones que surgían constantemente en el campo de la administración pública, hacían necesaria una organización que facilitara la recuperación de la información de manera rápida y eficaz. Esta biblioteca «especializada» venía acompañada de una planificación y de un reglamento. Las tres etapas de la planificación serían las siguientes:

$1^{\mathrm{a}}$.- Centralizar en una dependencia las diversas obras, revistas, folletos y demás impresos sobre Administración local que se encuentren dispersos en las diferentes dependencias de la Diputación.

$2^{\mathrm{a}}$.- Facilitar el acceso y estudio a las obras, a través de un meticuloso y ordenado fichero de materias.

$3^{\mathrm{a}}$.- Incrementar la biblioteca adquiriendo las obras más interesantes sobre temas técnicos y jurídicos relacionados con la Administración local. 
El resultado debía ser la publicación de un catálogo y de un índice de materias, que facilitara el manejo de la biblioteca, con sus sucesivos apéndices. Esta nueva biblioteca comportaba la incorporación de «personal de la casa con suficiente preparación para poder llevar a cabo el proyecto».

El Reglamento establecía que «El fin de la Biblioteca Técnica Provincial es dotar a la Excma. Diputación provincial de una dependencia bibliográfica, en la que los miembros de la Corporación y los funcionarios provinciales encuentren medios científicos y culturales adecuados, para el más perfecto desempeño de sus funciones». Además, se establecía que «en los locales de la biblioteca se reúnan todos los libros, impresos, mapas, manuscritos, etc. que, estando en diferentes dependencias, guarden relación con las materias de Administración local y con los servicios de la Administración Provincial. Formarán también parte de la biblioteca los boletines oficiales, gacetas y demás publicaciones periódicas».

Se acordaba la formación de un catálogo general de las obras, además de índices de materias, en el que se especificaba «muy especialmente en materia jurídicoadministrativa». También se establecían los criterios sobre conservación de la colección y la gestión de la misma, los servicios que debía prestar, etc. Al frente de la biblioteca y como responsable último debía estar el archivero-bibliotecario de la Diputación, bajo la dependencia directa de la Secretaría General.

Como disposición final se establecía que la biblioteca técnica funcionaría como una «Sección independiente dentro de la Biblioteca General de la Diputación cuando ésta se reorganice y reglamente formalmente». La biblioteca dejaba de denominarse provincial para convertirse en general y dentro de ésta se contemplaba que debía existir una sección especializada en temática legislativa. Fruto de esta biblioteca técnica es la interesante colección de legislación sobre las competencias que tenía la Diputación decimonónica, obras que actualmente forman parte de la biblioteca auxiliar del archivo de la Diputación y sirven de apoyo a los investigadores, entre las que caben destacar el diccionario de Alcubillas y un buen número de impresos referentes a ordenanzas y reglamentos. Por otra parte, también se contemplaba que la biblioteca general se transformara en una «Biblioteca Pública, tal como lo requiere la importancia de los fondos existentes y el prestigio cultural y buen nombre de la propia Corporación».

Con el paso de los años, la biblioteca siguió formando una misma unidad junto con el archivo y continuó incrementándose con obras editadas por la misma corporación, donaciones, intercambios y compras, ocupando uno de los torreones del Palacio de la Generalitat, hasta que en 1980 el archivo-biblioteca se trasladó a un nuevo emplazamiento: el edificio del Convento de la Trinidad, con acceso por la calle Alboraia.

Ese mismo año se producían cambios en la Institución Alfons el Magnànim. En la sesión de 3 de marzo de 1980 la corporación proponía a Josep Picó para el cargo de director de la Institución y se trasladaba la sede a la plaza de Alfonso el Magnánimo. Allí quedaba abierta al público una biblioteca que albergaba dos colecciones: la del 
Instituto Valenciano de Economía (IVE) y la «Saralegui» del Servicio de Estudios Artísticos, ambos institutos pertenecían a la Institució Alfons el Magnànim9 .

Poco tiempo después, se producían cambios de titularidad y de denominación: la Institució Alfons el Magnànim pasaba a denominarse Institució Valenciana d'Estudis i Investigació (IVEI) y se convertía en una entidad dependiente de dos organismos: Diputación y Generalitat. Una institución dedicada a promocionar y editar trabajos de investigación. En torno a ella, se fue gestando una importante biblioteca de apoyo a los investigadores. Tras la disolución de la IVEI, en 1997, esta biblioteca quedó arrinconada en unas dependencias de la Diputación hasta que en el año 2005 se hicieron las gestiones para incorporarla a la Biblioteca del MuVIM.

\section{Consolidación de la Biblioteca General de la Diputación}

En los primeros años de la década de los ochenta, mientras la Institució Alfons el Magnànim tomaba nuevos rumbos, el archivo-biblioteca se ubicaba en el nuevo espacio del Convento de la Trinidad y se procedía a la catalogación de la biblioteca. Poco después se jubilaba Arturo Zabala y comenzaba una nueva andadura bajo la dirección de Amparo García. El primer evento que se hizo en 1984, para dar a conocer la valiosa biblioteca de la Diputación, fue la exposición «Cervantes: Cuatro siglos en la Literatura Universal». La muestra fue organizada por el personal del archivo-biblioteca, siendo presidente de la Diputación Antonio Asunción, y diputado de cultura Jesús Ros, y se realizó en el edificio de la Lonja de Valencia, coincidiendo con la Feria del Libro. Fruto de la misma fue la edición del catálogo Cervantes: cuatro siglos en la literatura universal (Diputación de Valencia, 1984).

Un año después, en 1985, la Diputación adquiría una importante biblioteca privada, la «Biblioteca Alfons Roig». El padre Alfons Roig Izquierdo (1903-1987) donó su biblioteca y pinacoteca a la Diputación de Valencia. A lo largo de su vida este prócer de la cultura reunió casi 6.000 obras y más de 180 títulos de revistas. A través de su biblioteca se puede observar aquello que Josep Monter escribió sobre él: «el seu amor als llibres no era l'amor del bibliòfil, sinó el d'aquell que cerca i empra els llibres per a saber i entendre la pròpia vida i el propi món on viu: per això la seua biblioteca anava creixent al ritme de les preocupacions vitals dels seus moments històrics o, millor, existencials que, a més a més, anaven lligats íntimament amb els dels seus amics» (Monter, J., 2001).

Su gran pasión por el arte le llevó a relacionarse con artistas y críticos, a viajar por Europa e incluso a escribir sobre arte. El padre Roig recorrió España dando conferencias e introduciendo un poco de aire fresco europeo en una España de tinieblas, tal como rezaba una dedicatoria de María Zambrano: «Para Don Alfonso Roig, que admirablemente, en tiempos de impenetrable oscuridad, derramó palabras verdaderas con la obstinación del agua». Sus alumnos, tanto los de la escuela de Bellas Artes como los del Seminario lo recuerdan como la persona que, en la España de los años sesenta, les abrió los ojos al arte moderno, o mejor dicho, a lo moderno. De él escucharon, por primera vez, nombres de artistas como: Kandinsky, Mondrian, Henri 
Moore, Manessier etc.; les habló de libertad, de tolerancia, de escritores que vivían en el exilio.

Es un orgullo para la Biblioteca del MuVIM que su legado bibliográfico esté en ella, porque Alfons Roig fue para el siglo XX lo que los ilustrados lo fueron para el XVIII: el hombre que se atrevió a saber, el hombre que conoció, a través de la creación artística, los sentimientos y necesidades del mundo que le rodeaba, a entender la espiritualidad en el arte; a dar un paso adelante y despertar aquellos corazones sedientos de saber; a ellos les enseñó $L^{\prime}$ art viu del nostre temps ${ }^{10}$. Y, al igual que sus predecesores del siglo XVIII, fue muchas veces incomprendido y criticado. Tras la conferencia que dio sobre Picasso, a mediados de los años cincuenta, en la que mostraba públicamente su talante tolerante y religiosamente abierto, encontró un ambiente hostil. A través de las anotaciones en sus libros, sus comentarios y «sus papeles» podemos conocer mejor a ese hombre sencillo, humilde y culto. Su celo por guardar los catálogos y los trípticos de las exposiciones convierte este legado en fuente de primera mano para rastrear la actividad artística y expositiva de unos años difíciles para el arte moderno.

La Diputación siguió adquiriendo fondos para su biblioteca y en abril de 1989 compraba una colección de obras de teatro. La mayoría de estos impresos procedían de antiguos teatros valencianos, sobre todo del teatro Eslava. Durante la primera mitad del siglo XX estos teatros fueron centros neurálgicos de ocio cultural de la sociedad valenciana pero, poco a poco, fueron desapareciendo. Muchas de estas obras tienen el sello del teatro en la portada, un sello que si bien en su momento identificaba la propiedad de la obra, ahora nos informa de su procedencia. Se trata de una interesante colección de piezas teatrales con anotaciones manuscritas, en algunas de ellas, sobre variaciones de diálogos, cambios de personajes, decorados, etc.

Esta colección fue adquirida para completar la documentación del Teatro Principal, que se conserva en el archivo de la Diputación, y formar una interesante colección de obras teatrales que fueran la base de un futuro centro de documentación teatral. Se trata de una interesante colección formada por impresos de los siglos XVIII, XIX y primera mitad del XX, en la que cabe resaltar que algunas de estas obras están encuadernadas formando colecciones facticias. Otras, que formaban parte de un volumen facticio fueron desencuadernadas para ser vendidas sueltas. Esto ocurre sobre todo con los impresos del siglo XVIII, de los cuales existen gran número de obras anónimas, muchas de ellas identificadas como de Ramón de la Cruz (17311794).

Durante los años en que el archivo-biblioteca estuvo instalado en el edificio del Convento de la Trinidad se fueron realizando transferencias documentales de aquellas instituciones que habían dejado de existir: la Casa de la Misericordia y la Casa de Beneficencia, además de otras que seguían funcionando, como el Hospital General. Todo ello unido a las transferencias periódicas de las diferentes secciones de la Diputación. El espacio pronto quedó pequeño y se construyó un nuevo archivobiblioteca aprovechando parte del antiguo hospital psiquiátrico provincial «Padre 
Jofré», instalado tras la desamortización en el Convento de Jesús y trasladado en 1973 al nuevo hospital de Bétera.

Rehabilitado parte del edificio, se inauguraba el nuevo archivo-biblioteca en 1995, siendo presidenta de la Diputación, Clementina Ródenas. Dos años después se realizaba una exposición en el Centro Cultural La Beneficencia con el título «Cartografia valenciana: segles XVI-XIX». Para esta muestra se compraron mapas y planos que, una vez concluida la exposición, pasaron a formar parte de la biblioteca. Esta colección pasó a incrementar la ya existente colección cartográfica, con mapas editados por la misma Diputación, y en la cual también figuraba el Plano de Valencia del padre Tosca.

Este plano, grabado por el aragonés José Fortea en torno al año 1738, está basado en el plano original manuscrito que conserva el Ayuntamiento de Valencia y que Tomás Vicente Tosca (1651-1723) dibujó entre los años 1701 y 1704, reuniendo en su trazado un conjunto de conocimientos técnicos y matemáticos que demuestran la pujanza intelectual de los novatores valencianos del siglo XVII. Había sido adquirido por la Diputación en 1943, gracias a la donación realizada por Carlos Sarthou, tal como se desprende del expediente de la Comisión de Enseñanza, Cultura y Bellas Artes, de fecha 12 de marzo de 1943, en la cual se acuerda:

«[...] agradecer el nuevo donativo que para el Archivo-Biblioteca de esta Corporación ha efectuado el Cronista de Játiva, don Carlos Sarthou Carreres, consistente en un Plano de Valencia del Padre Tosca, de 1 x 1,50 M [ ...] y hacer presente a dicho señor, por su reiterada generosidad, un expresivo voto de gracias» ${ }^{11}$.

El mal estado de conservación en el que se encontraba el plano cuando se trasladó al MuVIM hizo que se gestionara su restauración. En el año 2007 el plano fue restaurado por el Institut Valencià de Conservació i Restauració de Bens Culturals ${ }^{12}$. Se digitalizó y actualmente está enmarcado y expuesto en la misma Biblioteca del MuVIM.

La Biblioteca general comenzaba una nueva andadura a finales del año 1999. Por Decreto de Presidencia $\mathrm{n}^{\circ} 10878$, de 16 de diciembre de 1999, se procedía a la reestructuración del área de cultura y por Acuerdo Plenario, de 22 de diciembre de 1999, la Corporación acordaba «el traslado de los fondos bibliográficos dependientes del Archivo-Biblioteca a la Red de Museos de la Corporación».

En el año 2001 se inauguraba el Museu Valencià de la Il·lustració i de la Modernidad y se procedía al traslado y organización de la biblioteca, siendo inaugurada en octubre de 2004. Desde ese momento la Biblioteca del MuVIM ha asumido, además de las funciones que le son propias como biblioteca de museo, las de ser biblioteca general de la Diputación, con funciones de recoger, conservar y difundir la producción editorial de la corporación provincial. 
Podemos concluir diciendo que la falta de un espacio físico destinado exclusivamente a biblioteca, unido a la diversificación de proyectos, hizo que la Biblioteca general de la Diputación no lograra una entidad propia. Cuando en el año 1976 el Ministerio de Cultura acordó crear una biblioteca pública provincial, dependiente del Estado, en los terrenos del antiguo hospital, la Biblioteca de la Diputación perdió la ocasión de poder convertirse en la biblioteca nacional valenciana. A partir de ese momento la presencia en la ciudad de Valencia de una Biblioteca Pública, inaugurada en 1979, alentó a la ciudadanía a participar en la conservación y difusión del patrimonio bibliográfico valenciano, siendo el primer legado el de Nicolau Primitiu Gómez Serrano, convirtiéndose en el germen de la futura Biblioteca Valenciana.

\section{Referencias bibliográficas y archivísticas:}

ARCHIVO GENERAL Y FOTOGRÁFICO DE LA DIPUTACIÓN DE VALENCIA (ADPV), Series documentales:

- ADPV, A.3.1.12. Memorias de la Diputación (1880-1980).

- ADPV, A.3.4.5. Expedientes generales del archivo (1980-2000).

- ADPV, E.8.2. Expedientes generales de Cultura (1900-1982).

- ADPV, E.9.1. Expedientes generales de Instrucción pública (1919-1940).

DÍAZ Y PÉREZ, N. (1885), Las bibliotecas en España en sus relaciones con la educación popular y la instrucción pública ( $2^{\mathrm{a}}$ ed.), Madrid.

DIPUTACIÓN DE VALENCIA (1984), Cervantes: cuatro siglos de literatura universal, Diputación de Valencia, Valencia.

DIPUTACIÓN DE VALENCIA (1999), La biblioteca Alfons Roig. Institució Alfons el Magnànim, Valencia.

GABARDA CEBELLÁN, V. (1997), Institución Alfons el Magnánimo. Institució Valenciana d'Estudis i Investigació (1947-1997), Edicions Alfons el Magnànim-IVEI, Valencia.

MONTER PÉREZ, J. (2001). "La biblioteca Alfons Roig”, Levante, el Mercantil valenciano, 5 de enero de 2001.

SÁNCHEZ-CUTILLAS, C. (1974), Francisco Martínez y Martínez, un humanista alteano (1866-1946), Caja de Ahorros Provincial, Alicante.

\section{Notas}

1. Los estatutos del Centro de Cultura Valenciana se publicaron en Anales del Centro de Cultura Valenciana. Tomo I, Año 1928, Valencia, p. 2. 
2. ARCHIVO DE LA DIPUTACIÓN DE VALENCIA (ADPV), Memoria...1920, p. 120.

3. ADPV, Memoria...1923, p. 45.

4. ADPV, Memoria...1925-26, p. 97.

5. ADPV, Memoria...1929, p. 74.

6. ADPV, Memoria ...1930, p. 99.

7. Durante la Guerra Civil el Consell Provincial Valencià acordó, en el Pleno del día 7 de febrero de 1937, la creación de l'Institut d'Estudis Valencians, con el objetivo de promocionar y difundir la cultura valenciana. Una de las principales aspiraciones de este instituto era crear la Biblioteca del País Valencià, proponiendo como fondo fundacional la Colección Cervantina. El cambio de régimen truncó este proyecto.

8. ADPV, E.8.2, c. 38, exp. 22.

9. En febrero de 1992 la «Colección Saralegui» fue incorporada a la Biblioteca del Museo de Bellas Artes San Pío V, donde ya había estado con anterioridad porque el «museo provincial» había sido sede del Servicio de Estudios Artísticos. El archivo y la biblioteca del IVE fueron cedidos temporalmente a la Biblioteca Valenciana en el año 2000.

10. Uno de los pocos libros que escribió Alfons Roig, publicado por la Diputación de Valencia en 1982.

11. ADPV, E.8.2/C.27, exp. 20.

12. Nuestro agradecimiento al personal del IVACOR que lo restauraron sin coste alguno para la corporación. 